\title{
EL PROYECTO MOMPA: PROTOCOLOS DE ACTUACIÓN BASADOS EN EL MONITOREO MEDIANTE INTERFEROMETRÍA SATELITAL RADAR (INSAR)
}

\section{MOMPA PROJECT: ACTUATION PROTOCOLS BASED ON SATELLITE RADAR INTERFEROMETRY (INSAR) MONITORING}

\author{
Anna Barra ${ }^{a}{ }^{,}$, Xavier Colell ${ }^{b}$, Anna Echeverria ${ }^{c}$, Laura Trapero ${ }^{c}$, Jordi Marturia ${ }^{d}$, Ivan Fabregat ${ }^{d}$, Qi

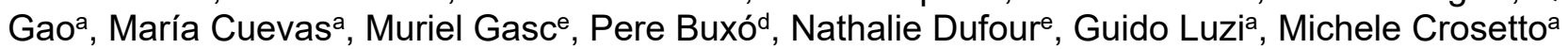 \\ a Centre Tecnològic de Telecomunicacions de Catalunya (CTTC/CERCA), Av. Carl Friedrich Gauss 7, 08860 Castelldefels, España. \\ anna.barra@cttc.es; qi.gao@cttc.es; maria.cuevas@cttc.es; gluzi@cttc.cat; michele.crosetto@cttc.es \\ b Euroconsult, Carrer de Na Maria Pla, 33, AD500, Andorra la Vella, Andorra. xcolell@euroconsult.ad
}

c Snow and Mountain Research Center of Andorra (CENMA-IEA), Institut d'Estudis Andorrans, Av. Rocafort 21-23, Sant Julià de Lòria, Andorra. aecheverria@iea.ad; Itrapero@iea.ad

d Institut Cartogràfic i Geològic de Catalunya (ICGC), Parc de Montjuïc, 08038 Barcelona, España. Jordi.Marturia@icgc.cat; Ivan.Fabregat@icgc.cat; Pere.Buxo@icgc.cat

e Centre d'études et d'expertise sur les risques, l'environnement, la mobilité et l'aménagement (CEREMA), 1 Avenue du Colonel Roche, 31400 Toulouse, Francia.muriel.gasc@cerema.fr; nathalie.dufour@cerema.fr

\begin{abstract}
:
The objective of the POCTEFA - MOMPA project (Monitoring of Ground Movements and Action Protocol) is to provide a useful technical-operational tool for the prevention and management of risks due to slope movements, at a cross-border level, based on the satellite monitoring technique InSAR (Synthetic Aperture Radar Inteferometry). The tool includes two main elements: the risk evaluation, and the integration of the technique in an action protocol for the administrations in charge of Civil Protection. The study area covers around $4,000 \mathrm{~km}^{2}$ and includes a part of the eastern Pyrenees, including the countries of Spain, France, and Andorra. The project deals with risk assessment based on the ground displacement map at an interregional scale and on the detected Active Deformation Areas (ADA), to select movements with potential risk where it will then focus on an analysis at a local scale using traditional methods (photo interpretation and field work). Both the free medium-resolution data, acquired by the Sentinel-1 satellite, as well as the high-resolution data acquired by COSMO-SkyMed are used. In addition, the project focuses on the landslide event of "la Portalada" (in Andorra) that occurred in 2019. Today the slope shows a slow movement that could affect a main road located at the bottom of the valley. The Government of Andorra is implementing measures to monitor and characterize the current movement of the slope. Therefore, nine artificial reflectors were installed along this forested slope. The data obtained will be integrated into the risk prevention protocol. Here, the project and the first results obtained through satellite interferometry are presented.
\end{abstract}

Key words: MOMPA, POCTEFA, deformation maps, InSAR, Pyrenees, landslides

\section{Resumen:}

El objetivo del proyecto POCTEFA - MOMPA (MOnitorización de Movimientos del terreno y Protocolo de Actuación) es proporcionar una herramienta técnico-operativa útil para la prevención y la gestión de los riesgos ocasionados por movimientos de ladera, a nivel transfronterizo, basada en la técnica de seguimiento satelital InSAR. (Interferometría Radar a Apertura Sintética) La herramienta incluye dos elementos principales: la evaluación del riesgo; y la integración de la técnica en un protocolo de actuación para las administraciones encargadas de la Protección Civil. El área de estudio cubre alrededor de $4.000 \mathrm{~km}^{2}$ e incluye una parte de los Pirineos orientales, incluyendo los países España, Francia y Andorra. El proyecto trata la evaluación de riesgos a partir del mapa de movimientos del terreno a escala interregional y de las Áreas de Deformación Activa (ADA), para seleccionar movimientos con riesgo potencial donde luego se enfocará en un análisis a escala local mediante el uso de métodos tradicionales (básicamente fotointerpretación y trabajo de campo). Se utilizan tanto los datos libres y gratuitos de resolución media, adquiridos por el satélite Sentinel-1, como los datos de alta resolución adquiridos por COSMO-SkyMed. Además, el proyecto centra su atención en el caso particular del deslizamiento de "la Portalada" (Andorra) ocurrido en 2019. En la actualidad, la ladera presenta un movimiento lento que podría afectar el eje principal de comunicación entre Andorra y España ubicado en el fondo del valle. El Gobierno de Andorra está implementando medidas para monitorear y caracterizar el movimiento actual de la ladera. Por tanto, nueve reflectores artificiales se instalaron a lo largo de esta ladera ocupada por un bosque. Los datos obtenidos se integrarán en el protocolo de prevención de riesgos. En este trabajo se presentan el proyecto y los primeros resultados obtenidos mediante interferometría satelital.

*Corresponding Author: Anna Barra, anna.barra@cttc.es 
Palabras clave: MOMPA, POCTEFA, Mapas de deformación del terreno, InSAR, Pirineos, deslizamientos

\section{Introducción}

La gestión de los riesgos a escala regional se basa principalmente en mapas de peligrosidad, o de susceptibilidad, que se basan en el conocimiento del territorio y en datos históricos de eventos. Estos mapas son muy útiles, pero son generalmente informaciones estáticas, que se actualizan cada vez que un nuevo evento ocurra. Además, los elevados costes de la monitorización in situ de los movimientos del terreno condicionan a que la gestión de los riesgos geológicos asociados sea frecuentemente de tipo "reactivo", es decir se enfoca solamente en los movimientos del terreno evidentes, de alto riesgo, o que ya han generado daños.

El objetivo de MOMPA es mejorar la gestión del riesgo, contribuyendo a pasar de una aproximación "reactiva" a una "proactiva". El elemento innovador del proyecto es la técnica de interferometría de imágenes radar de apertura sintética (InSAR), basada en imágenes satelitales para la mejora de la gestión de los riesgos geológicos. Esta técnica aprovecha la diferencia de fase entre imágenes SAR adquiridas en la misma área, en tiempos diferentes. Las diferencias de fase se utilizan para estimar posibles movimientos de la superficie del terreno ocurridos durante el período de tiempo cubierto por las fechas de adquisiciones. La técnica nos permite obtener millones de mediadas de movimientos, sobre amplias áreas (escala regional) con una precisión de orden milimétrico (Massonnet and Feigl 1998). Esto nos permite de un lado una detección y actualización periódica de los movimientos a escala regional, de otro lado la posibilidad de monitorizar y caracterizar el comportamiento temporal de varios movimientos, sin necesidad de medidas en situ. Una revisión de los métodos avanzados de interferometría satelital radas se proporciona en Crosetto et al. 2016.

En las últimas décadas, el mapeo y monitoreo de fenómenos de peligro geológico han recibido una importante contribución de las técnicas InSAR. Algunos ejemplos de aplicación son: actividad volcánica (Peltier et al. 2010; Bonforte et al. 2011; Lagios et al. 2013), movimientos tectónicos (Massironi et al. 2009; Vilardo et al. 2010; Lagios et al. 2012), hundimientos (Tomás et al. 2014; Solari et al. 2016; Béjar-Pizarro et al. 2017) o caracterización y monitoreo de deslizamientos de tierra (Notti et al. 2010; Herrera et al. 2013; Oliveira et al. 2015; Barra et al. 2016; Reyes-Carmona et al. 2020; Solari et al. 2020). Es evidente que existe una integración de la técnica InSAR en la gestión de los riesgos geológicos como herramienta de detección y monitoreo. Sin embargo, salvo pocas excepciones de utilizo integrado en la gestión regional de riesgos (Bianchini et al. 2018; Raspini et al. 2019; Montalti 2019), su uso se ha limitado al ámbito científico debido a su dificultad de procesado y de interpretación. La puesta en funcionamiento de constelaciones con políticas de adquisición sistemática y acceso gratuito a los datos (como Sentinel-1) ha supuesto un incremento en el interés por estas técnicas $y$ un enfoque en el desarrollo de herramientas y procedimientos para incrementar el utilizo operativo en la gestión del territorio (Barra et al. 2017, 2018, Del Soldato, 2019, Bianchini et al. 2020).
En este contexto, el proyecto MOMPA quiere introducir la interferometría satelital en la gestión de los riesgos en un área de los pirineos orientales, proponiendo un enfoque interregional y piramidal (de pequeña a grande escala) basado en la interferometría satelital.

Después de una presentación general del proyecto (sección 2) y de la zona de estudio (sección 2.1), se presentarán los primeros resultados logrados con el uso de la técnica InSAR con imágenes adquiridas por los satélites Sentinel-1 y COSMO-SkyMed (sección 3). Así mismo, se presentan los resultados preliminares de un estudio de un deslizamiento de interés, donde se instalaron 9 reflectores artificiales serán comentados en la sección 4 .

\section{El proyecto MOMPA}

El proyecto MOMPA (MOnitorización de Movimientos del terreno y Protocolo de Actuación) está cofinanciado por el Fondo Europeo de Desarrollo Regional (FEDER) a través del Programa Interreg V-A España-FranciaAndorra (POCTEFA 2014-2020). POCTEFA 2014-2020 es un programa europeo de cooperación territorial creado para fomentar el desarrollo sostenible del territorio fronterizo entre los tres países (Fig. 1) (POCTEFA 2021).

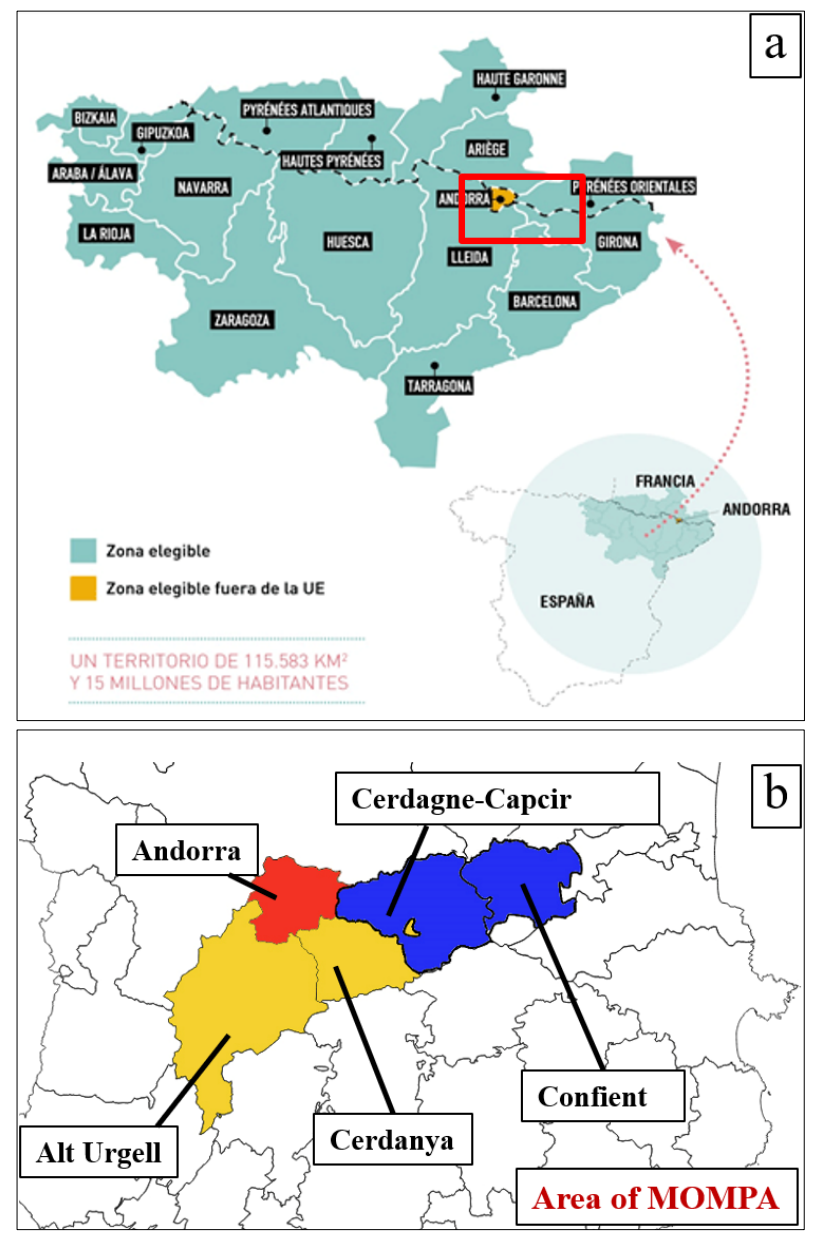

Figura 1: Zona de estudiol: a) zona elegible del programa POCTEFA; b) área de estudio del proyecto MOMPA: en azul la zona francesa, en amarillo la zona española, y en rojo la andorrana. 
MOMPA se enmarca en el eje estratégico 2 del programa "Promover la adaptación al cambio climático y la prevención y gestión de riesgos", y con el objetivo específico de "Mejorar la capacidad de anticipación y respuesta de los actores del territorio a los riesgos específicos y a la gestión de catástrofes naturales".

El proyecto se inició el 1 de diciembre de 2019 y finalizará en mayo de 2022 (MOMPA 2021). El partenariado incluye cuatro socios especializados en teledetección y en técnicas de análisis y gestión del riesgo: el Centre Tecnològic de Telecomunicacions de Catalunya (CTTC), el Institut d'Estudis Andorrans (IEA), el Institut Cartogràfic i Geològic de Catalunya (ICGC), y el Centre d'études et d'expertise sur les risques, l'environnement, la mobilité et l'aménagement (CEREMA). Cuenta como asociados las protecciones civiles de cada territorio y la empresa Euroconsult Andorra S.A..

Los resultados principales esperados del proyecto son los siguientes: (i) mapas de seguimiento de los movimientos del terreno en una zona incluida en los territorios de l'Alt Urgell, Cerdanya, Capcir, Conflent y Andorra; (ii) valoraciones de riesgo asociado; (iii) protocolos de actuación de soporte a la Protección Civil. Se trata de resultados permanentes, en línea con la estrategia Europa 2020, que serán transferidos a las Protecciones Civiles (socios asociados) y a otras organizaciones, como las Autoridades locales y regionales.

La idea del proyecto es tener un enfoque piramidal en la gestión de los riesgos: a partir de los mapas de deformación a escala interregional, se detectan las Áreas de Deformación Activa (ADA) y se clasifican en función del nivel de riesgo potencial asociado. En las ADA de interés se hace un estudio de más detalle, priorizando la inversión de recursos, tiempo y análisis (integrando otras técnicas y datos), en función del nivel potencial de riesgo asociado a cada ADA.

Después de las acciones de preparación, gestión y comunicación del proyecto, las principales acciones técnicas son:

- Acción 3 - Monitorización de los movimientos del terreno: realización de mapas de velocidad anual de deformación y series temporales de deformación. Extracción de las Áreas de Deformación Activa (ADA) detectadas. Se utilizan dos tipos de datos: media (Sentinel-1) y alta resolución (COSMOSkyMed).

- Acción 4 - Análisis de riesgo asociada a movimientos del terreno: valoración de riesgo de los elementos expuestos en base a una delimitación de los fenómenos geológicos asociados a las ADA detectadas en la Acción 3. Se prevé realizar dos análisis: el primero se basará en los mapas de deformación y las series temporales de deformación a media resolución, el segundo se basará en los datos a alta resolución.

- Acción 5 - Protocolo de actuación: realización de dos actividades principales: el análisis de los umbrales de prealerta o alerta de los movimientos analizados, y el desarrollo metodológico de los protocolos de actuación para la Protección Civil.

\section{1. Área de estudio}

Geográficamente, la zona de estudio del proyecto MOMPA cubre un área de aproximadamente $4.000 \mathrm{~km}^{2}$, e incluye las siguientes regiones de los Pirineos centroorientales (Fig. 1): Alt Urgell y Cerdanya, en España, Cerdagne-Capcir y Conflient, en Francia, y el principado de Andorra. Los movimientos del terreno que pueden comportar riesgo son diversos principalmente deslizamientos y flujos, caída de rocas, y subsidencias.

Los datos de teledetección son muy útiles y recomendados en la gestión de deslizamientos de tierra (Mantovani et al. 1996; Metternicht et al. 2005; Tofani et al. 2013; Scaioni et al. 2014). Entre estos, el uso de productos interferométricos para estudios de deslizamientos de tierra lo describe bien Wasowski and Bovenga (2014), destacando las ventajas y limitaciones de la técnica. La zona de los Pirineos no presenta características favorables a la aplicación de la técnica InSAR, y por eso no existen muchos trabajos previos (Herrera 2011). La zona de estudio presenta características que limitan la aplicación de la técnica, así como explicado en Wasowski and Bovenga (2014). Los principales factores limitantes de la AOI son:

- Cobertura del suelo: la zona de estudio está cubierta mayoritariamente por vegetación: bosques, prados y áreas agrícolas. La vegetación genera una respuesta del suelo inestable a la señal RADAR, lo que implica ruido $\mathrm{o}$ imposibilidad de obtener medidas.

- Cobertura de nieve: las zonas más elevadas de la zona de estudio están cubiertas por un manto de nieve durante muchos días al año. Esto implica que las imágenes adquiridas en días con nieve no se puedan utilizar si se quiere obtener un resultado global en zonas de montaña.

- Topografía: la fuerte topografía implica una geometría relativa suelo-adquisición muy variable en el espacio. Al ser un sistema que solo puede medir movimientos en la dirección LOS, la línea de vista del satélite, la capacidad de detectar el movimiento depende de su dirección real. Además, la pendiente del terreno define la huella o resolución espacial de nuestro píxel: en una zona como la de MOMPA, la resolución varía mucho entre zonas.

\subsection{Acción 3: Monitorización de los movimientos del terreno}

Esta sección describe las primeras actividades de la Acción 3 del proyecto MOMPA, enfocada en el utilizo de InSAR para la monitorización de los movimientos del terreno. Esta acción representa una componente fundamental del proyecto, ya que sus resultados son los datos principales de entrada de las Acciones 4 y 5 .

La monitorización de los movimientos de la Acción 3 se basa en la técnica InSAR, que usa una serie de imágenes radar, de tipo SAR, adquiridas desde satélites. Es importante destacar que la técnica InSAR no funciona de forma homogénea sobre el territorio. De hecho, la técnica funciona bien sobre zonas urbanas, infraestructuras, zonas rocosas, etc., mientras que puede tener una densidad de medidas menor, o hasta ninguna medida, en zonas con mucha vegetación (Hanssen 2005). 
Las estimaciones de las deformaciones a partir de las imágenes SAR se ha hecho utilizando el software y procesado PSIG, adaptada a la zona de estudio. Para más detalles ver Biescas et al. (2007); Crosetto et al. (2016) y Devanthéry et al. (2014).

Esta acción prevé dos tipos de análisis InSAR: una a media y otra a alta resolución.

\subsubsection{Procesado de Media resolución}

Los datos de media resolución $\left(4 \times 14 \mathrm{~m}^{2}\right)$ son adquiridos por los satélites Sentinel-1, en geometría descendente. Son datos gratuitos y que permiten cubrir grandes áreas gracias al método de adquisición Wide Swath (IWS). En consecuencia, toda la zona de interés de MOMPA cabe en el marco de una sola imagen (Fig. 2). En el especifico se han generado los interferogramas a partir de seis bursts (subimágenes) de dos swaths (Fig. 2) de las imágenes Sentinel-1 A y B. Para el primer mapa de deformación, se han procesado un total de 203 imágenes que cubren el período comprendido entre el 4 de julio de 2015 hasta el 28 de diciembre de 2020.

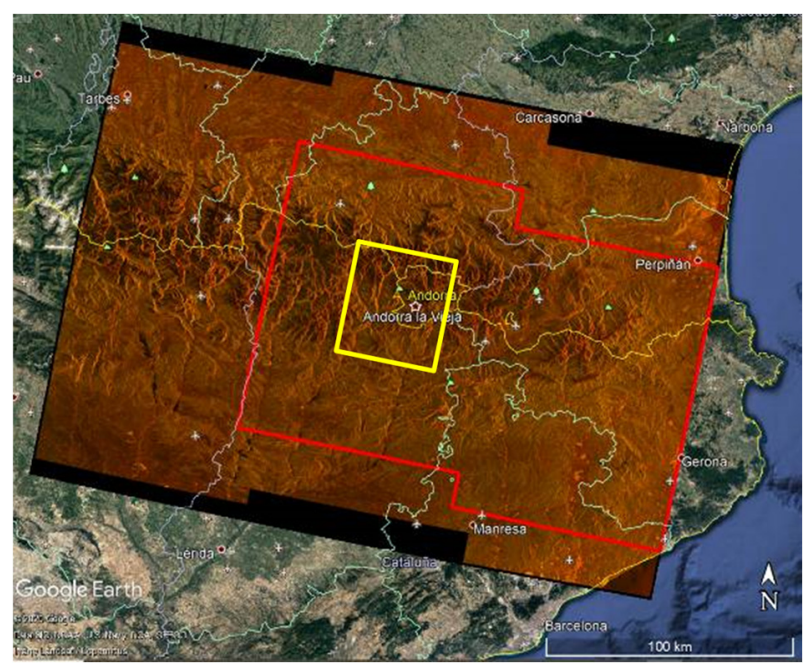

Figura 2: En color marrón se representa la extensión de las imágenes Sentinel-1. El polígono rojo indica la parte de la imagen que ha sido procesada para la generación de los interferogramas. El polígono amarillo indica la extensión de las imágenes COSMO-SkyMed. Imagen de fondo de Google Earth.

Las imágenes, descargadas a través del portal Copernicus Open Access Hub, tienen en general, una separación temporal de 6 o 12 días. No obstante, algunas imágenes de los periodos invernales (diciembre-mayo) han sido eliminadas para evitar problemas de coherencia debida a la cobertura de nieve (Solari et al. 2020; Mirmazloumi et al. 2021). Estos datos nos han permitido obtener el mapa de velocidades sobre toda la zona de estudio (Fig. 3) utilizando el procesado PSIG explicado en Biescas et al. (2007) y Devanthéry et al. (2014).

A partir de los mapas globales de velocidades se han extraído las áreas de deformación activas (ADA) de manera automática con la utilización de la herramienta ADAFinder (Barra et al. 2017; Tomás et al. 2019; Navarro et al. 2020). Las áreas extraídas facilitan la interpretación y la utilización de los productos InSAR, ayudan a enfocar las actividades de análisis a las zonas de mayor interés.

Gracias a la detección de las ADA, se han seleccionado áreas de mayor interés por su potencial riesgo. Sobre estas áreas, en manera local, se han generado las series temporales de deformación con el método explicado en Devanthéry et al. (2014).

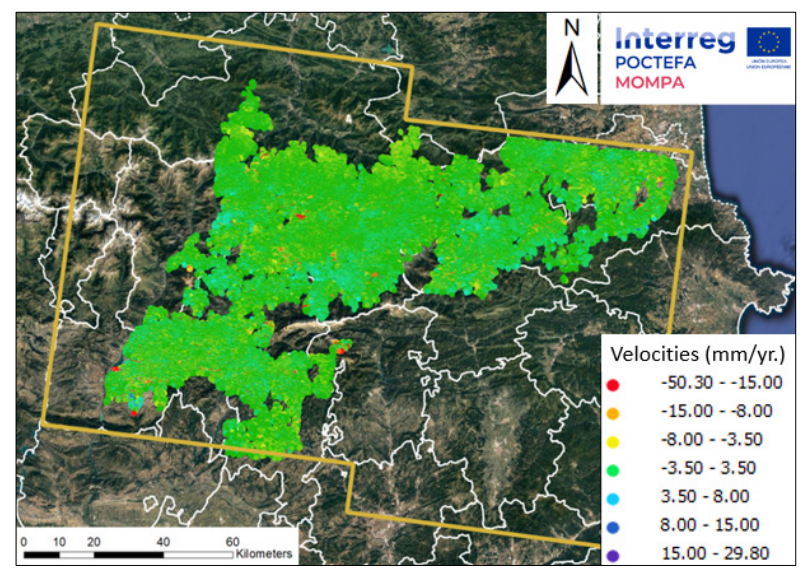

Figura 3: Mapa de velocidades de deformación (mm/año) estimada a partir de datos de media resolución Sentinel-1.

\subsubsection{Procesado de alta resolución}

Los datos de alta resolución $\left(3 \times 3 \mathrm{~m}^{2}\right)$ son adquiridos por los satélites Cosmo-SkyMED (de Telespazio Iberica), en modo de adquisición Stripmap, con geometría descendente. El marco de las imágenes seleccionadas (marcado en amarillo en la Figura 2) cubre toda Andorra y la parte norte del Alt Urgell. Para los primeros resultados de alta resolución, se han procesado 36 imágenes disponibles que cubren un periodo comprendido entre Julio de 2013 a y Julio de 2020. Se ha generado un mapa de velocidades anuales de deformación cubriendo los principales núcleos urbano. A partir de estos resultados será posible extraer las ADA de alta resolución, y será posible un análisis de riesgo de más detalle, en las zonas urbanas.

\subsubsection{Monitorización con reflectores}

El 10 de agosto de 2019 el principal acceso a Andorra desde Catalunya, a través de la Seu d'Urgell, fue cortado por la ocurrencia de un gran desprendimiento (Fig. 4). El incidente sucedió a las seis de la mañana en la Portalada (Sant Julià de Lòria), en una zona en obras junto a un importante centro comercial. El desprendimiento obligó a cerrar el tráfico en ambos sentidos. Las autoridades andorranas reabrieron la circulación en los dos sentidos de la marcha de la carretera CG-1, entre Andorra y La Seu d'Urgell, después de más de 6 horas.

El deslizamiento de ladera de la Portalada, inducido probablemente por las excavaciones realizadas al lado de la carretera, es el fenómeno conocido más importante en el territorio cubierto por el proyecto MOMPA. Por esta razón, se decidió analizar en detalle esta zona usando la técnica InSAR. Tal y como se describe a continuación, el estudio InSAR de la zona requiere el despliegue de una red de reflectores artificiales.

La técnica InSAR explota la respuesta de objetos (reflectores) a las microondas de los sensores SAR, que tiene que ser persistente en el tiempo. Normalmente las zonas cubiertas por vegetación tienen muy pocos reflectores. 


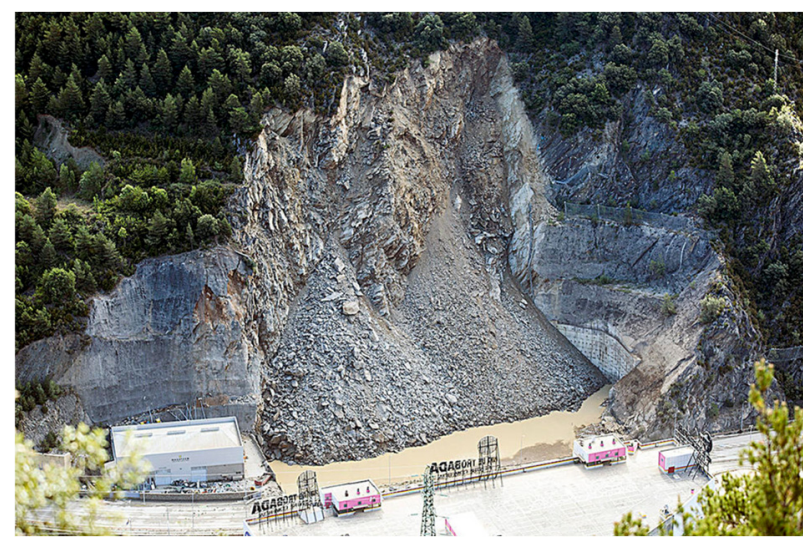

Figura 4: Deslizamiento de la Portalada (Andorra) del 10/08/2019. Fuente: Diari d'Andorra (2021).

Este es el caso de la zona de la Portalada donde: la ladera bajo monitoreo está a ladera arriba del desprendimiento del 2019 y está cubierta por bosque (Fig. 5), lo que implica la ausencia de reflectores naturales que nos permitan adquirir medidas con la técnica InSAR. Por este motivo, y por el alto interés de la zona, se ha elegido instalar nueve reflectores artificiales (ocho pasivos y uno activo, Figs. 5 y 6 ) que aseguran una reflexión estable de la signar radar permitiendo obtener medidas de movimientos (Crosetto et al. 2013; Luzi et al. 2021). La primera instalación de la red de reflectores se finalizó el 1 de diciembre de 2020 gracias al apoyo de las administraciones locales. El primer objetivo de cualquier red de reflectores es comprobar la respuesta de cada reflector con un análisis de la amplitud, y luego derivar las primeras estimaciones de los desplazamientos. Los reflectores están orientados según la geometría de adquisición de los datos Sentinel-1 utilizados, para aprovechar la alta frecuencia de adquisiciones (una imagen cada 6 días).

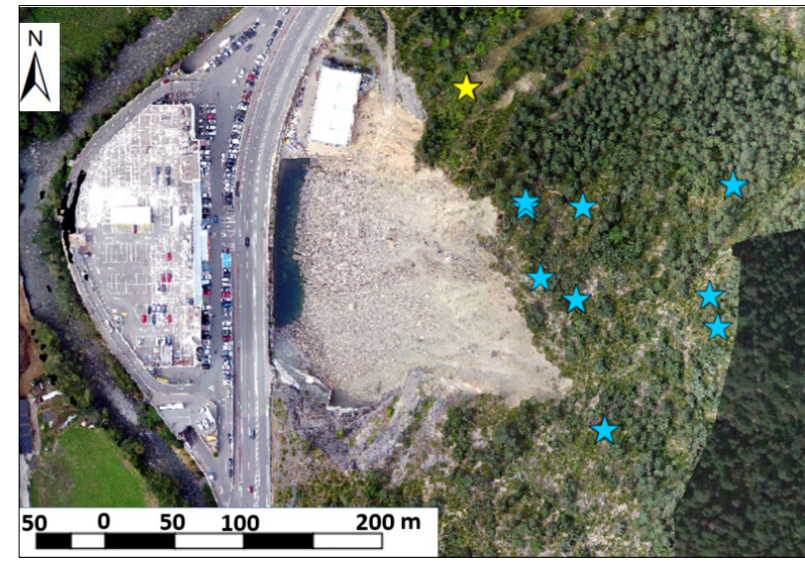

Figura 5: Posición de la red de reflectores. Las estrellas azules indican la posición de los reflectores pasivos. La estrella amarilla indica la posición del reflector activo.

\section{Resultados}

La Figura 3 presenta el mapa global de velocidades de deformación, estimado con una solución única sobre la totalidad del área del proyecto, donde se puede destacar una sensibilidad media de $3.5 \mathrm{~mm} / a n ̃ o$. En la Figura $7 \mathrm{se}$ muestra un ejemplo de un deslizamiento localizado en Andorra, medido con ambos datos de media resolución
(Fig. 7a) y alta resolución (Fig. 7b). Ambos resultados describen muy bien la extensión del deslizamiento.

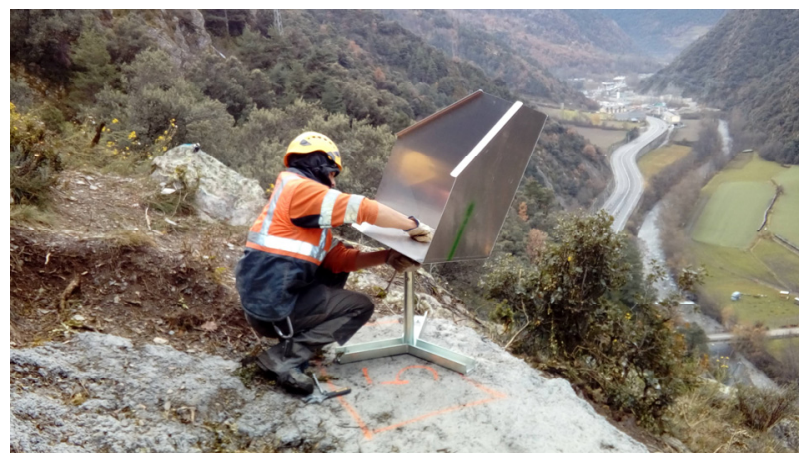

Figura 6: Instalación y orientación de un reflector pasivo en la ladera en movimiento de La Portalada (Andorra, diciembre de 2020).
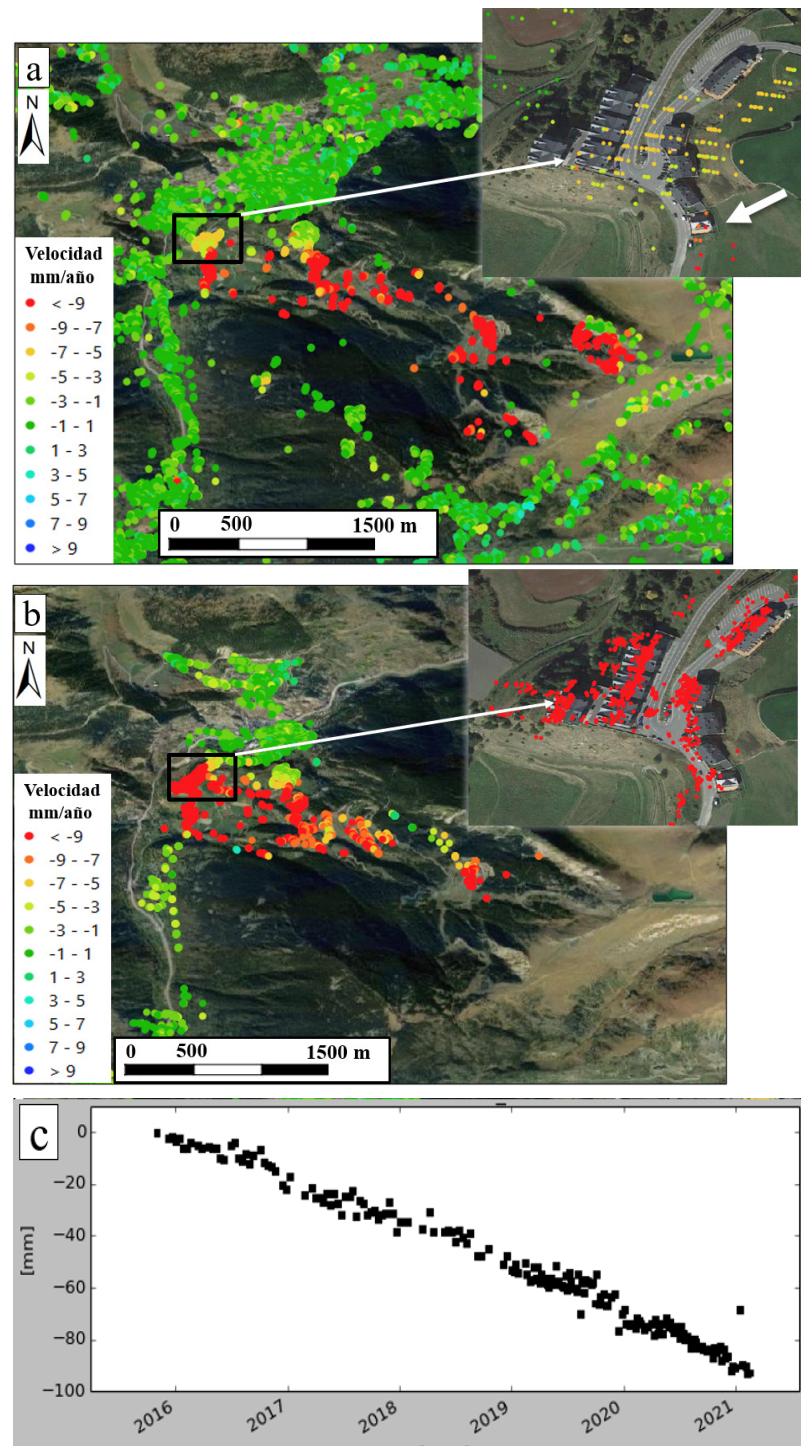

Figura 7: Ejemplo de una zona en deslizamiento, detectada en ambos procesados de media (a) y alta (b) resolución. En (c) se muestra un ejemplo de serie temporal de deformación estimada a partir de datos Sentinel-1 de media resolución.

Es notorio que los datos a mayor resolución (es decir con menor longitud de onda) tienen peor coherencia en zonas de cambio. Se puede destacar como Sentinel-1 ofrece 
una cobertura más completa y extensa debido a su mayor coherencia sobre zonas vegetadas. Mientras que los datos de alta resolución son más sensibles al ruido debido por ejemplo a la cobertura vegetal (Crosetto et al. 2010). Por otro lado, observando los dos detalles de la Figura 7, es evidente como CosmoSkyMed, ofrece un muestreo muy detallado (de aproximadamente un punto cada 3 por $3 \mathrm{~m} 2$ ), en correspondencia con los edificios, estructuras e infraestructuras. La Figura 7c muestra un ejemplo de serie temporal de deformación de un punto localizado en la zona señalada en la Figura 7a, estimada a partir de los datos Sentinel-1.

\subsection{Monitorización de la Portalada (Andorra) con reflectores}

En esta fase del proyecto, se ha evaluado la respuesta, en términos de amplitud de las imágenes SAR, de diferentes pixeles, con el objetivo de seleccionar los pixeles que se corresponden a los reflectores y de individuar posibles problemas. Se considera que un reflector está funcionando bien cuando el píxel correspondiente tiene un incremento permanente de amplitud justo después de la instalación del mismos (los reflectores se instalaron el 1 diciembre de 2020). La serie temporal de la Figura 8 contiene la secuencia de amplitudes de un mismo píxel donde la mayoría de los valores están entre 0 y 100 . Sin embargo, hay una fecha (el 1 de diciembre de 2020) donde estos valores suben a 255 (bytes) y después se mantienen constantes: Se trata claramente de un reflector artificial, concretamente del reflector activo marcado con 1 en la Figura 9. Gracias a este análisis se ha individuado un reflector que no estaba dando señal y se ha podido individuar el problema con una inspección de campo: el reflector no estaba correctamente orientado.

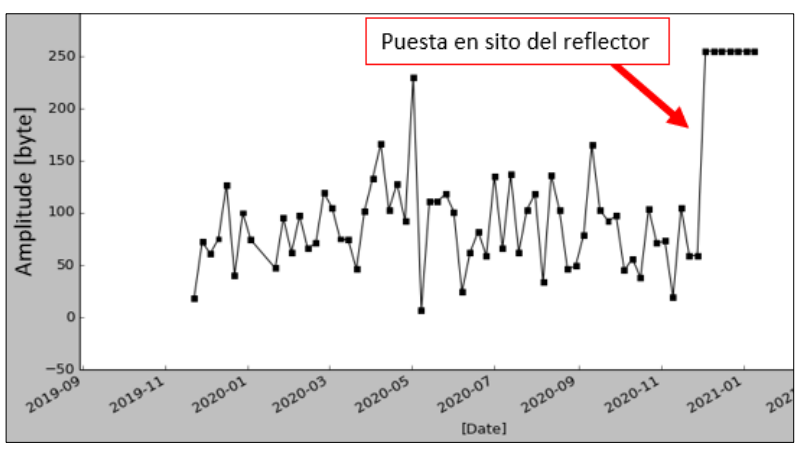

Figura 8: Serie temporal de amplitud del reflector activo.

Una vez individuados los reflectores en las imágenes, se han estimado las series temporales de deformación sobre los puntos seleccionados (Figs. 8 y 10). Las series de deformación se han estimado a partir de la primera imagen disponible después de la instalación de los reflectores: incluye 7 imágenes desde el 04/12/2020 hasta el 09/01/2021. La Figura 9 ilustra un mapa de deformación acumulada de la zona de interés. Se puede observar que en la zona del centro comercial (parte izquierda de la Figura 9) hay una buena respuesta con muchos puntos, la mayoría de los cuales son estables (color verde). Es importante observar que en la parte derecha de la Figura 9 aparecen grupos de puntos (p. ej. dos, tres o más puntos). Dichos puntos se corresponden típicamente a un único reflector, cuya respuesta representa múltiples píxeles. Normalmente se usa el punto con mayor amplitud de respuesta y se descartan los otros.

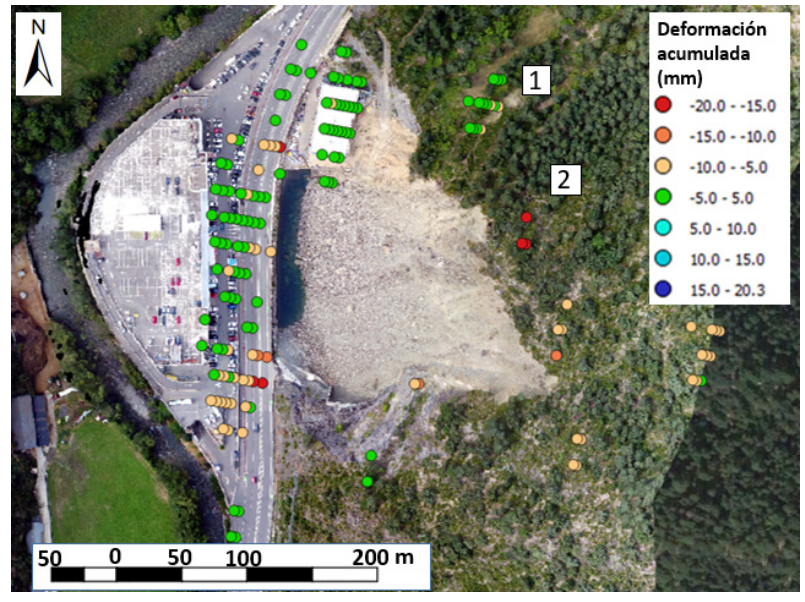

Figura 9: Resultado preliminar de la red de reflectores y de otros reflectores de la zona. Los colores indican la deformación acumulada.

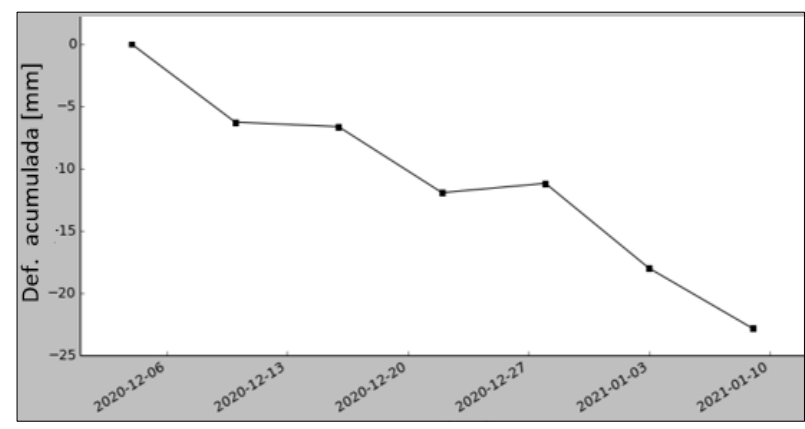

Figura 10: Serie temporal de deformación del reflector pasivo indicado con el número 2 en la Figura 9.

En la Figura 10 se muestra la serie temporal de deformación del reflector señalado con el número 2 en la Figura 9. Con este primer resultado podemos detectar un movimiento post-deslizamiento que afecta la ladera arriba del desprendimiento. Además, la zona que presenta la serie temporal enseñada (Figs. 8 y 10) se ha movido el más o menos el doble de los otros reflectores. Esos movimientos son importantes de monitorizar para ver si están decelerando o no en el tiempo. Los resultados se analizarán juntos con otros datos de monitorización in situ.

\section{Conclusiones}

En este trabajo se ha descrito el proyecto MOMPA con un enfoque sobre la Acción 3, que incluye el procesado InSAR de media y alta resolución. Se han presentado unos primeros resultados que han permitido seleccionar las áreas de interés para las siguientes acciones del proyecto, dedicadas a un análisis de riesgo y caracterización de los movimientos. Los resultados mostrados serán la base para desarrollar protocolos de actuación útiles para los servicios de protección civil, o las autoridades involucradas en la gestión del riesgo. Esta información será de utilidad para actuar en función de la información derivada por la técnica de interferometría radar de satélite. Los resultados serán transferidos a las Protecciones Civiles (socios asociados 
del proyecto) y a otras administraciones públicas locales y regionales.

Se ha presentado un mapa de velocidades de deformación que cubre integralmente la zona de estudio del proyecto, que incluye Alt Urgell y Cerdanya en Cataluña, Capcir, Conflent en Francia y Andorra. Con una zona de interés relacionada a un movimiento detectado en Andorra se ha mostrado en detalle un ejemplo de medidas derivadas por ambos procesados de media (datos Sentinel-1) y alta (datos Cosmo-SkyMED) resolución. Este ejemplo permite una primera comparación entre los dos resultados. Al contrario de Sentienl-1, los datos de alta resolución no aseguran adquisiciones regulares en el tiempo, y no son gratuitos. Esto implica de un lado que no se puede planificar un monitoreo a largo plazo, de otro lado implica una dificultad (a veces imposibilidad) de derivar las series temporales de deformación. Otro aspecto es la mayor sensibilidad a las deformaciones de un lado positivo, y al ruido de un lado negativo. Esto nos ha implicado una dificultad alta en obtener resultados integrados en toda la zona, debido a la baja densidad de urbanización. Hasta ahora podemos afirmar que en la gestión regional de los riesgos los datos de alta resolución no resultan necesarios y no son favorables a la operatividad a largo plazo, mientras la utilización de datos Sentinel-1 son más que satisfactorios. De otro lado, a escala de mayor detalle en os elementos antrópicos, nos permiten un estudio de detalle como por ejemplo detectar edificios afectados por mayores movimientos diferenciales y entonces sujetos a un deño potencial mayor.

Además, se han presentado los resultados preliminares de la zona de estudio de La Portalada, después de la instalación de los reflectores artificiales. El valor añadido del empleo de una red de reflectores artificiales en esta zona de interés será evaluado durante el curso del proyecto.

\section{Agradecimientos}

Este trabajo ha sido co-financiado por el Fondo Europeo de Desarrollo Regional ("European Regional Development Fund") a través de Interreg V-A España, Francia y Andorra (POCTEFA 2014-2020), proyecto EFA295/19. En el caso de la Portalada, los autores agradecen a la administración local de La Portalada, el Comú de Sant Julià de Lòria, por permitir la implementación de los reflectores, y al Ministerio de Ordenación Territorial del Gobierno de Andorra por facilitar su gestión. La obra de implementación de los reflectores fue contratada a la empresa INACCÉS.

\section{References}

BARRA, A., MONSERRAT, O., MAZZANTI, P., ESPOSITO, C., CROSETTO, M., and SCARASCIA, G., 2016. First insights on the potential of Sentinel-1 for landslides detection. Geomat Nat Haz Risk, 7, pp. 1-10

BARRA, A., MONSERRAT, O., SOLARI, L., HERRERA, G., LOPEZ, C., ONORI, R., REICHENBACH, P., GONZÁLEZALONSO, E.L.E.N.A., MATEOS, R.M., BIANCHINI, S., and CROSETTO, M., 2018. The safety project: SENTINEL-1 for the management of geological risk. WIT Transactions on Engineering Sciences, 121, pp. 247-258.

BARRA, A., SOLARI, L, BÉJAR-PIZARRO, M., MONSERRAT, O., BIANCHINI, S., HERRERA, G., CROSETTO, M., SARRO, R., GONZÁLEZ-ALONSO, E., MATEOS, R.M., LIGÜERZANA, S., LÓPEZ, C., and MORETTI, S., 2017. A Methodology to Detect and Update Active Deformation Areas Based on Sentinel-1 SAR Images. Remote Sensing, 9(10), 1002 .

BÉJAR-PIZARRO, M., EZQUERRO, P., HERRERA, G., TOMÁS, R., GUARDIOLA-ALBERT, C., HERNÁNDEZ, JMR., FERNANDEZ MERODO, JA., MARCHAMALO, M., and MARTÍNEZ, R., 2017. mapping groundwater level and aquifer storage variations from InSAR measurements in the Madrid aquifer, central Spain. $j$ hydrol., 547 pp. 678-689

BIANCHINI, S., RASPINI, F., SOLARI, L., DEL SOLDATO, M., CIAMPALINI, A., ROSI, A., and CASAGLI, N., 2018. From picture to movie: Twenty years of ground deformation recording over Tuscany region (Italy) with satellite InSAR. Frontiers in Earth Science, 6, p.177.

BIANCHINI, S., SOLARI, L., BARRA, A., MONSERRAT, O., CROSETTO, M., and CATANI, F., 2020. Sentinel-1 PSI Data for the Evaluation of Landslide Geohazard and Impact. In Workshop on World Landslide Forum (pp. 447-455). Springer, Cham.

DIARI D'ANDORRA. 2021. Diari d'Andorra (diariandorra.ad). Available: https://www.diariandorra.ad/ [7/15, 2021].

BIESCAS, E., CROSETTO, M., AGUDO, M., MONSERRAT, O., and CRIPPA, B., 2007. Two radar interferometric approaches to monitor slow and fast land deformation. Journal of Surveying Engineering, 133(2), pp.66-71.

BONFORTE, A, GUGLIELMINO F, COLTELLI M, FERRETTI A, and PUGLISI G., 2011. structural assessment of mount etna volcano from permanent scatterers analysis. Geochem Geophy Geosy. 12, pp. 1-19

CROSETTO, M., MONSERRAT, O., IGLESIAS, R., and CRIPPA, B., 2010. Persistent scatterer interferometry. Photogrammetric Engineering \& Remote Sensing, 76(9), pp.1061-1069.

CROSETTO, M., GILI, J.A., MONSERRAT, O., CUEVAS-GONZÁLEZ, M., COROMINAS, J., and SERRAL, D., 2013. Interferometric SAR monitoring of the Vallcebre landslide (Spain) using corner reflectors. Natural Hazards and Earth System Sciences, 13(4), pp. 923-933.

CROSETTO, M., MONSERRAT, O., CUEVAS-GONZÁLEZ, M., DEVANTHÉRY, N., and CRIPPA, B., 2016. Persistent Scatterer Interferometry: A review. ISPRS J. Photogramm. Remote Sensing, 115, pp. 78-89. 
DEL SOlDATO, M., SOLARI, L., RASPINI, F., BIANCHINI, S., CIAMPALINI, A., MONTALTI, R., FERRETTI, A., PELLEGRINESCHI, V., and CASAGLI, N., 2019. monitoring ground instabilities using sar satellite data: a practical approach. ISPRS International Journal of Geo-Information, 8(7), p.307.

DEVANTHÉRY, N., CROSETTO, M., MONSERRAT, O., CUEVAS-GONZÁLEZ, M., and CRIPPA, B., 2014. An approach to persistent scatterer interferometry. Remote Sensing, 6(7), pp. 6662-6679.

HANSSEN, R.F., 2005. satellite radar interferometry for deformation monitoring: a priori assessment of feasibility and accuracy. International Journal Of Applied Earth Observation And Geoinformation, 6(3-4), pp.253-260.

HERRERA, G., NOTTI, D., GARCÍA-DAVALILLO, J.C., MORA, O., COOKSLEY, G., SÁNCHEZ, M., ARNAUD, A., and CROSETTO, M., 2011. analysis with c-and x-band satellite SAR data of the Portalet landslide area. Landslides, 8(2), pp.195-206.

HERRERA, G., GUTIÉRREZ, F., GARCÍA-DAVALILLO, J.C., GUERRERO, J., NOTTI, D., GALVE, J.P., FERNANDEZMERODO, J.A., and COOKSLEY, G., 2013. multi-sensor advanced DInSAR monitoring of very slow landslides: the Tena Valley case study (central Spanish Pyrenees). Remote Sens Environ., 128, pp. 31-43

LAGIOS, E., PAPADIMITRIOU, P., NOVALI, F., SAKKAS, V., FUMAGALLI, A., VLACHOU, K., and DEL CONTE, S., 2012. combined seismicity pattern analysis, DGPS and PSInSAR studies in the broader area of Cephalonia (Greece). Tectonophysics, 524, pp. 43-58

LAGIOS, E., SAKKAS, V., NOVALI, F., BELLOTTI, F., FERRETTI, A., VLACHOU, K., and DIETRICH, V., 2013. SqueeSAR ${ }^{\mathrm{TM}}$ and GPS ground deformation monitoring of Santorini volcano (1992-2012): tectonic implications. Tectonophysics, 594, pp. 38-59.

LUZI, G., ESPÍN-LÓPEZ, P.F., MIRA PÉREZ, F., MONSERRAT, O., and CROSETTO, M., 2021. A Low-Cost Active Reflector for Interferometric Monitoring Based on Sentinel-1 SAR Images. Sensors, 21(6), 2008.

MANTOVANI, F., SOETERS, R., and VAN WESTEN, C.J., 1996. Remote sensing techniques for landslide studies and hazard zonation in Europe. Geomorphology, 15, pp. 213-225. DOI: 10.1016/s0148-9062(97)87261-0

MASSIRONI, M., ZAMPIERI, D., BIANCHI, M., SCHIAVO, A., and FRANCESCHINI, A., 2009. use of PSInSAR ${ }^{\mathrm{TM}}$ data to infer active tectonics: clues on the differential uplift across the Giudicarie Belt (central-eastern Alps, Italy). Tectonophysics, 476, pp. 297-303

MASSONNET, D., and FEIGL, K. L., 1998. Radar interferometry and its application to changes in the Earth's surface. Rev. Geophys., 36, pp. 441-500. DOI: 10.1029/97RG03139

METTERNICHT, G., HURNI, L., and GOGU, R., 2005. Remote sensing of landslides: An analysis of the potential contribution to geo-spatial systems for hazard assessment in mountainous environments. Remote Sens Env., 98, pp. 284-303. DOI: 10.1016/j.rse.2005.08.004

MIRMAZLOUMI, S.M., BARRA, A., CROSETTO, M., MONSERRAT, O., and CRIPPA, B., 2021. Pyrenees deformation monitoring using sentinel-1 data and the persistent scatterer interferometry technique. Procedia Computer Science, 181, pp.671-677.

MOMPA. 2021. MOnitorización de Movimientos del terreno y Protocolo de Actuación. Home . Available: https://mompa.eu/es/ [7/15, 2021].

MONTALTI, R., SOLARI, L., BIANCHINI, S., DEL SOLDATO, M., RASPINI, F., and CASAGLI, N., 2019. A Sentinel-1based clustering analysis for geo-hazards mitigation at regional scale: a case study in Central Italy. Geomatics, Natural Hazards and Risk, 10(1), pp. 2257-2275.

NAVARRO, J. A., TOMÁS, R., BARRA, A., PAGÁN, J. I., REYES-CARMONA, C., SOLARI, L., VINIELLES, J. L., FALCO, S., and CROSETTO, M., 2020. ADAtools: Automatic Detection and Classification of Active Deformation Areas from PSI Displacement Maps. ISPRS Int. J. Geo-Inf., 9(10), p. 584.

NOTTI, D., DAVALILLO, J.C., HERRERA, G., and MORA O., 2010. assessment of the performance of x-band satellite radar data for landslide mapping and monitoring: upper Tena Valley case study. Nat Hazards Earth Sys., 10, pp. 1865

OLIVEIRA, S.C., ZÊZERE, J.L., CATALÃO, J., and NICO, G., 2015. the contribution of PSInSAR interferometry to landslide hazard in weak rock-dominated areas. Landslides, 12(4), pp.703-719.

PELTIER, A., BIANCHI, M., KAMINSKI, E., KOMOROWSKI, J.C., RUCCI, A., and STAUDACHER, T., 2010. PSInSAR as a new tool to monitor pre-eruptive volcano ground deformation: validation using GPS measurements on piton de la Fournaise. Geophys Res Lett., 37, pp. 1-5.

POCTEFA. 2021. ¿Qué es POCTEFA?. Available: https://www.poctefa.eu/programa/que-es-poctefa/\# [7/15, 2021].

RASPINI, F., BIANCHINI, S., CIAMPALINI, A., DEL SOLDATO, M., MONTALTI, R., SOLARI, L., TOFANI, V., AND and CASAGLI, N., 2019. persistent scatterers continuous streaming for landslide monitoring and mapping: the case of the Tuscany region (Italy). Landslides, 16(10), pp.2033-2044. 
REYES-CARMONA, C., BARRA, A., GALVE, J.P., MONSERRAT, O., PÉREZ-PEÑA, J.V., MATEOS, R.M., NOTTI, D., RUANO, P., MILLARES, A., LÓPEZ-VINIELLES, J., and AZAÑÓN, J.M., 2020. sentinel-1 DInSAR for monitoring active landslides in critical infrastructures: the case of the Rules reservoir (southern Spain). Remote Sensing, 12(5), p. 809.

SCAIONI, M., LONGONI, L., MELILLO, V., and PAPINI, M., 2014. Remote sensing for landslide investigations: an overview of recent achievements and perspectives. Remote Sens., 6, pp. 9600-9652. DOI: 10.3390/rs6109600

SOLARI, L., CIAMPALINI, A., RASPINI, F., BIANCHINI, S., and MORETTI, S., 2016. PSInSAR analysis in the Pisa urban area (Italy): a case study of subsidence related to stratigraphical factors and urbanization. Remote Sens., 8, p. 120.

SOLARI, L., BIANCHINI, S., FRANCESCHINI, R., BARRA, A., MONSERRAT, O., THUEGAZ, P., BERTOLO, D., CROSETTO, M., and CATANI, F., 2020. Satellite interferometric data for landslide intensity evaluation in mountainous regions. International Journal of Applied Earth Observation and Geoinformation, 87, p. 102028.

SOLARI, L., DEL SOLDATO, M., RASPINI, F., BARRA, A., BIANCHINI, S., CONFUORTO, P., CASAGLI, N., and CROSETTO, M., 2020. review of satellite interferometry for landslide detection in Italy. Remote Sens., 12, p. 1351.

TOFANI, V., SEGONI, S., AGOSTINI, A., CATANI, F., and CASAGLI, N., 2013. Use of remote sensing for landslide studies in Europe. Nat Hazards Earth Syst Sci., 13, pp. 299-309. DOI: 10.5194/nhess-13-299-2013

TOMÁS, R., ROMERO, R., MULAS, J., MARTURIÀ, J.J., MALLORQUÍ, J.J., LÓPEZ-SÁNCHEZ, J.M., HERRERA, G., GUTIÉRREZ, F., GONZÁLEZ, P.J., FERNÁNDEZ, J., and DUQUE, S., 2014. Radar interferometry techniques for the study of ground subsidence phenomena: a review of practical issues through cases in Spain. Environ Earth Sci., 71, pp. 163-181.

TOMÁS, R., J. PAGÁN, J. I., NAVARRO, J.A., CANO, M., PASTOR, J. L., RIQUELME, A., CUEVAS-GONZÁLEZ, M. CROSETTO, M., BARRA, A., MONSERRAT, O., LÓPEZ-SÁNCHEZ, J. M., RAMÓN, A., IBORRA, S., DEL SOLDATO, M., SOLARI, L., BIANCHINI, S., RASPINI, F., NOVALI, F., FERRETI, A., CONSTANTINI, M., TRILLO, F., HERRERA, G., and CASAGLI, N., 2019. Semi-Automatic Identification and Pre-Screening of Geological-Geotechnical Deformational Processes Using Persistent Scatterer Interferometry Datasets. Remote Sensing, 11(14), p. 1675.

VILARDO, G., ISAIA, R., VENTURA, G., DE MARTINO, P., and TERRANOVA, C., 2010. InSAR permanent scatterer analysis reveals fault re-activation during inflation and deflation episodes at Campi Flegrei caldera. Remote Sens Environ., 114, pp. 2373-2383

WASOWSKI, J., and BOVENGA, F., 2014. Investigating landslides and unstable slopes with satellite Multi Temporal Interferometry: Current issues and future perspectives. Eng Geol., 174, pp. 103-138. DOI: 10.1016/j.enggeo.2014.03.003 